OPEN ACCESS

Edited by:

Claire Dumas,

Institut National de la Recherche

Agronomique (INRA), France

Reviewed by:

Robert Heyer,

Medizinische Fakultät,

Universitätsklinikum Magdeburg,

Germany

Shengguo Zhao,

Institute of Animal Science (CAS),

China

*Correspondence:

Natalie Ferry

n.ferry@salford.ac.uk

Specialty section:

This article was submitted to

Systems Microbiology,

a section of the journal

Frontiers in Microbiology

Received: 30 June 2017 Accepted: 24 October 2017 Published: 08 November 2017

Citation:

Joynson R, Pritchard L, Osemwekha E and Ferry N (2017) Metagenomic Analysis of the Gut Microbiome of the

Common Black Slug Arion ater in

Search of Novel Lignocellulose Degrading Enzymes.

Front. Microbiol. 8:2181.

doi: 10.3389/fmicb.2017.02181

\section{Metagenomic Analysis of the Gut Microbiome of the Common Black Slug Arion ater in Search of Novel Lignocellulose Degrading Enzymes}

\author{
Ryan Joynson ${ }^{1,2}$, Leighton Pritchard ${ }^{3}$, Ekenakema Osemwekha ${ }^{1}$ and Natalie Ferry ${ }^{1 *}$ \\ ${ }^{1}$ School of Environment and Life Science, University of Salford, Greater Manchester, United Kingdom, ${ }^{2}$ Earlham Institute, \\ Norwich, United Kingdom, ${ }^{3}$ Information and Computational Sciences, James Hutton Institute, Dundee, United Kingdom
}

Some eukaryotes are able to gain access to well-protected carbon sources in plant biomass by exploiting microorganisms in the environment or harbored in their digestive system. One is the land pulmonate Arion ater, which takes advantage of a gut microbial consortium that can break down the widely available, but difficult to digest, carbohydrate polymers in lignocellulose, enabling them to digest a broad range of fresh and partially degraded plant material efficiently. This ability is considered one of the major factors that have enabled $A$. ater to become one of the most widespread plant pest species in Western Europe and North America. Using metagenomic techniques we have characterized the bacterial diversity and functional capability of the gut microbiome of this notorious agricultural pest. Analysis of gut metagenomic community sequences identified abundant populations of known lignocellulose-degrading bacteria, along with well-characterized bacterial plant pathogens. This also revealed a repertoire of more than 3,383 carbohydrate active enzymes (CAZymes) including multiple enzymes associated with lignin degradation, demonstrating a microbial consortium capable of degradation of all components of lignocellulose. This would allow A. ater to make extensive use of plant biomass as a source of nutrients through exploitation of the enzymatic capabilities of the gut microbial consortia. From this metagenome assembly we also demonstrate the successful amplification of multiple predicted gene sequences from metagenomic DNA subjected to whole genome amplification and expression of functional proteins, facilitating the low cost acquisition and biochemical testing of the many thousands of novel genes identified in metagenomics studies. These findings demonstrate the importance of studying Gastropod microbial communities. Firstly, with respect to understanding links between feeding and evolutionary success and, secondly, as sources of novel enzymes with biotechnological potential, such as, CAZYmes that could be used in the production of biofuel.

Keywords: CAZymes, lignocellulose, Arion ater, biofuel, shotgun metagenomics, whole genome amplification, cellulase 


\section{INTRODUCTION}

Slugs are a highly successful group of organisms that are members of the order Pulmonata, found in high abundance in many terrestrial and aquatic ecosystems worldwide. The common black slug, Arion ater, is particularly prevalent in Western Europe and North America. These slugs travel long distances at night feeding on a variety of foodstuffs including vegetation (both live and decaying), carrion, and fungi. They use a tonguelike appendage containing barb-like teeth - the radula - to shred their food into uniformly sized pieces, increasing the surface area for enzymatic degradation in the gut. These slugs feed actively down to temperatures approaching $0^{\circ} \mathrm{C}$, and adults and eggs have been observed to survive freezing at $-3^{\circ} \mathrm{C}$ for 3 days or more (Slotsbo et al., 2011). It is therefore believed that slugs survive seasonal weather either by preservation of buried eggs or through migration to areas unaffected by frosts, such as, deep in compost heaps and underground in leaf litter (Kozlowski, 2007). Slugs are also known to be resistant to high concentrations of toxic metals, so much so that they are often used in studies of environmental levels of pollution (Ireland, 1979; Seric Jelaska et al., 2014). The ability to utilize a broad range of food sources and their physiological robustness to environmental challenges are amongst the reasons why slugs are such a successful group of organisms, despite the best efforts of humans to eradicate them from agricultural and suburban land.

It is now well established that the gut microbiome plays a pivotal role in digestion in many invertebrates and vertebrates such as, termites (Brune, 2014), cockroaches (Bertino-Grimaldi et al., 2013), cattle (Hess et al., 2011), and humans (Qin et al., 2010). However, the gut microbial communities of members of the gastropod class are still largely unstudied, despite their ability to digest a wide range of materials efficiently. One recent study has demonstrated the ecological richness of the gut microbiome of the gastropod Achatina fulica (giant snail), highlighting its metabolic capabilities, with a large number of CAZymes being observed (Cardoso et al., 2012a). In a previous study we demonstrated that the gut microbial consortium of A. ater is directly involved in breakdown of the lignocellulose portion of its diet (Joynson et al., 2014), while showing that this enzymatic activity is stable at a broad range of temperatures and $\mathrm{pH}$ levels. This suggests that the gut environment of $A$. ater could harbor microbial consortia of considerable ecological and economic importance.

In this study, we examine the composition of gut microbial consortia in A. ater, and their metabolic capability. There are three reasons why this research is important. First, knowledge of the gut microbiome composition of A. ater offers a means of understanding how this microbial population may facilitate the digestion of lignocellulose along with identification of a large number of CAZymes of interest to many industries including development of second generation biofuels. Second, it may offer insight into the survivability and feeding ability of slug species. This is especially important now, following the European Union ban on traditional molluscicide pellets, in force from September 2014 (Commission Implementing Regulation 187/2014), which was introduced because of the rapid build-up of molluscicide metabolites in water sources (Kay and Grayson, 2013). Finally, the microbiological profile of the slug gut may also provide a target for future bacterial crop pathogen diagnostics, tracking, and control measures in agriculture. Slugs have recently been proposed as vectors for the transmission of bacterial pathogens (Gismervik et al., 2014) and the metabolic capacity of soft rotting pathogens such as, Dickeya spp. (identified in this study) and many others could be advantageous in the mollusc gut (Toth et al., 2011).

\section{MATERIALS AND METHODS}

\section{Sample Collection and Metagenomics DNA Extraction}

Slugs were collected from a suburban area in North Cheshire $(53.391463 \mathrm{~N}, 2.211214 \mathrm{~W})$, a sampling area used in a previous study (Joynson et al., 2014), $2 \mathrm{~h}$ after last light. Individuals were cooled to $4^{\circ} \mathrm{C}$ to reduce spontaneous mucus production during dissection. Whole gut tracts were extracted, and care was taken to avoid rupturing the gut wall, to minimize loss or contamination of gut juices. All dissections were carried out in a sterile petri dish. Ten gut tracts were then pooled and DNA extracted using a modified protocol based on the MetaG-Nome DNA isolation kit (Epicentre, WI, USA). Briefly, gut pieces were homogenized in an extraction buffer by vortexing, and a series of centrifugation steps were then carried out to remove plant material from the gut and other large debris. Supernatants were then filtered through a $1.2 \mu \mathrm{m}$ filter in order to capture eukaryotic cell debris followed by a microbe capture step using a $0.2 \mu \mathrm{m}$ filter. Microbes were then washed off the filter and DNA was extracted. DNA quality and quantity was assessed spectrophotometrically (260:230 and 260:280 nm ratios) and using agarose gel electrophoresis alongside a pre-quantified fosmid control. Extracted DNA was then used to create an Illumina DNA library and sequenced using a Miseq using the V2 chemistry $(2 \times 250 \mathrm{bp})$ at the Centre for genomic research, Liverpool University.

\section{Metagenome Assembly, Functional/Phylogenetic Analysis}

Reads with ambiguous bases, along with their respective pair read, were removed from the raw dataset. Adaptor sequences were then trimmed from raw reads. Sequence output files were assessed using FastQC version 0.10.01 (Andrews, 2010). 25,996,846 reads passed quality control according to FastQC defaults and were assembled using Velvet (V1.2.10) (Zerbino and Birney, 2008) using options: $k=51$, cov_cutoff $=$ auto, exp_cov $=$ auto and ins_length 200. Velvet output de Bruijn graphs were then used as input to Metavelvet (v1.2.01) (Namiki et al., 2012). To assess the quality of the resulting assembly, raw reads were aligned to resultant contigs using (Burrow-Wheeler Aligner) BWA with default settings (Li et al., 2009). The resulting SAM file was then converted to a.BAM file, sorted, indexed, and mapping statistics obtained using the Samtools (Li et al., 2009) view, sort, index, and flagstat functions respectively. The resulting BAM file was visualized using the TABLET alignment viewer (Milne 
et al., 2013) facilitating manual curation during selection of novel genes for amplification and biochemical assay. Assembly output contigs were then subjected to open reading frame prediction using the $a b$ initio gene prediction method of MetaGeneMark (Zhu et al., 2010). Amino acid sequence files were then used as queries in a BLAST search against the NCBI nr protein database (03/2014) using options: $E$-value cutoff of $1 \mathrm{E}^{-5}$, num_alignments 50 , and num_descriptions 50 in order to assign putative function. The BLAST alignments were then used to organize predicted proteins into function and phylogeny using MEGAN4 (Huson et al., 2011). The lowest common ancestor (LCA) algorithm of MEGAN4 was used to sort open reading frame alignments into taxonomic groups using default parameters. For functional assignment, the predicted genes were sorted into groups based on the BLAST alignment results and the biochemical pathways annotated in the KEGG database using the KEGG extension in the MEGAN4 software. Further functional assignment was made by searching the predicted proteins against the CAZy database (Lombard et al., 2014). To do this all predicted sequences were used as a query in the CAZYmes Analysis Toolkit (CAT) (Park et al., 2010) using the Pfam based annotation tool with an Evalue threshold of $\times 10^{-4}$. Further phylogenetic analysis was carried out by subjecting raw sequencing reads to analysis using MetaPhlAn V1.7.8 (Segata et al., 2012) incorporating BowTie2 (Langmead and Salzberg, 2012). Raw reads were also uploaded to the MG-RAST pipeline (Meyer et al., 2008) for functional and taxonomical assignment along with estimation of taxonomic abundance. SEED analysis was used to compare the functional repertoire of slug gut microbiome against public MG-RAST gut metagenomes for higher termites (Costa Rican Nasutitermes sp.) cattle (Bos taurus), the Asian longhorn beetle (Anoplophora glabripennis) and the giant African land snail (A. fulica). In order to gain insight into biologically meaningful and statistically significant differences between the functional capacities of the slug gut and other microbiomes, the two-way Fisher's exact test with Benjamin-Hochberg FDR multiple test correction analysis was carried out pair wise between SEED annotations of the slug gut microbiome and those of comparator organisms using Statistical Analysis of Metagenomic Profiles (STAMP) (Parks et al., 2014). A. ater sequencing data was submitted to EBI ENA database (project ID: PRJEB21599).

\section{Amplification, Cloning, and Expression of CAZymes}

To increase the amount of metagenomic DNA template available for metagenome validation and amplification of identified genes, metagenomic DNA from the same sample that was used in sequencing was subjected to whole genome amplification (WGA). Ten nanogram of metagenomic sample DNA was used as template for amplification using the Repli-G mini kit (Qiagen, Manchester, UK), producing 4-6 $\mu \mathrm{g}$ of whole genome amplified product per $10 \mathrm{ng}$ starting material. In order to validate the metagenomic assembly a selection of predicted CAZY gene sequences were amplified using $100 \mathrm{ng}$ of WGA metagenomic DNA as template using Taq based PCR. PCR products were separated using $1 \%$ agarose gel electrophoresis and bands of sizes corresponding to the size of the predicted genes were gel extracted. PCR primer sequences and predicted genes sizes can be found in Supplementary Dataset 3. Amplified bands were then cloned and transformed into E. coli using the TA cloning kit (pCR2.1 vector) (Invitrogen). Vector inserts were sequenced using the BigDye 3.1 system to confirm CAZyme identity. One full length gene was subsequently re-amplified using Taq based PCR and cloned into the PBAD TOPO TA expression vector (Life Technologies, Paisley, UK). Proteins were expressed according to the manual instructions, and expressed products assessed using western blot targeting a $\mathrm{C}$ terminal His-tag. Detection was carried out using a secondary antibody-HRP conjugate and the ECL prime chemiluminescence kit (GE healthcare, Buckinghamshire, UK).

\section{CAZymes Activity Detection}

To detect enzyme functionality, transformed strains expressing proteins were then grown on agar activity assay plates. Strains containing predicted $\beta$-glucosidase cloned pBAD TOPO TA expression vectors were induced as per manual instructions. Five micro liter of induced culture was grown on LB agar plates containing $0.1 \%(\mathrm{w} / \mathrm{v})$ of the cellobiose mimic, esculin hydrate (Sigma, UK), and $0.03 \%(\mathrm{w} / \mathrm{v})$ ferric ammonium citrate (Sigma, UK) for $24 \mathrm{~h}$. The production of black halos was taken to indicate $\beta$-glucosidase activity. Untransformed TOP10 E. coli was used as a negative control.

\section{RESULTS}

\section{Metagenomic Library Sequencing}

Metagenomic DNA isolated from the whole gut tract, including crop and stomach was successfully extracted and the purity and genomic integrity tested as described. Sequencing of the metagenomic DNA yielded over $6 \mathrm{Gbp}$ of raw sequence data in the form of $\sim 26$ million paired-end reads, with an average length of $238 \mathrm{bp}$. The resulting community metagenome contained $81.74 \mathrm{Mbp}$ of sequence data with assembled contigs having an N50 value of $1.8 \mathrm{Kbp}$ (Table 1). This metagenome was then mined to determine the gut community ecology profile, along with the functional and metabolic capabilities of the microbiome.

\section{A. ater Gut Microbial Diversity}

Metagenomic community analysis showed that bacterial DNA predominated in the sample, with $99.4 \%$ of reads corresponded to bacteria, and only $0.3 \%$ to viruses, $0.2 \%$ to eukaryotes, and $0.01 \%$ to archaea (Supplementary Dataset 1). This suggests that attempts to limit the number of host and plant DNA contaminants by filtering was highly successful. Relative abundance of microbial groups was assessed using MetaPhlAn. This analysis indicated that the majority of the gut microbial community corresponded to members of the Gammaproteobacteria class (82\%) with most assignments being to members of the Enterobacteriaceae (64.5\%) and Pseudomonadaceae (10.6\%) families, which both contain widespread environmentally-adapted bacteria. Other families with notably high representation in the gut were Sphingobacteriaceae (8.6\%), Moraxellaceae (3.7\%), and 
TABLE 1 | Sequencing and assembly statistics of the gut community metagenome.

\begin{tabular}{lc}
\hline & A. ater Gut Metagenome \\
\hline Number of trimmed reads & $25,996,846$ \\
Raw sequence data (Gbp) & 6.175 \\
Number of assembled contigs & 48,089 \\
Largest contig (Kbp) & 56.3 \\
N50 value (Kbp) & 1.8 \\
Protein coding genes & 108,691 \\
Total size of metagenome (Mbp) & 81.74 \\
\hline
\end{tabular}

Flavobacteriaceae (1.8\%). The most abundant genera found in the gut microbiome were Enterobacter (26.9\%), Citrobacter (19.8\%), Pseudomonas (10.5\%), Escherichia (3.9\%), and Acinetobacter (3.6\%), and the genera Pantoea (2.7\%), Klebsiella (2\%), Serratia (0.75\%), Erwinia (0.73\%), and Salmonella (1.1\%) were also identified at lower abundance (Table 1, Figure 1, Supplementary Dataset 2). In order to compare the assignments and abundance data generated here, reads were also submitted to the MG-RAST pipeline which uses global alignments in its analysis unlike the marker gene database system used by MetaPhlAn. The MG-RAST pipeline produced results comparable to those from MetaPhlAn; again, the Gammaproteobacteria class was by far the most numerous in the sample, with the majority of those hits matching the Enterobacter family (Supplementary Dataset 1).

\section{Presence of Potential Plant Pathogens}

To determine the presence of plant pathogen species harbored in the $A$. ater gut, the metagenome phylogenetic analysis results were mined for hits relating to known plant pathogen species using the phylogenetic analyses of MataPhlAn and MG-RAST. Multiple assignments of metagenome sequence to plant pathogenic bacteria could be made, and Table 3 shows six economically significant plant pathogens identified in the A. ater gut microbiome. These include the three most economically-damaging bacterial crop pathogens in Europe: Erwinia amylovora, Dickeya dadanttii, and Pectobacterium carotovorum. (The species in Table 3 were identified by both MetaPhlan and MG-RAST analysis methods).

\section{Functional Analysis and Bacterial Metabolic Processes}

In order to assess the biochemical/metabolic potential of the gut microbiome, genes were predicted from assembled contigs. In total 108,691 putative genes were identified. These predictions were translated into amino acid sequences and used as queries for protein family identification, based on hits to the CAT Pfam database. This search identified 2,510 genes corresponding to glycoside hydrolase activity and 561 carbohydrate-binding modules. The majority of the carbohydrate-active genes identified were assigned to enzyme groups that break oligosaccharides down into simple sugars $(641,20.8 \%)$, with fewer targeting cellulose (26 enzymes, $0.85 \%$ ) (Table 4). This search also identified 312 members of the relatively new CAZyme classes "Auxiliary activities" or AA classes, which describes enzyme classes that act on or consort with lignin in their activities (Levasseur et al., 2013). This included 150 members of the class AA3, 2 members of AA2, 11 members of AA4, which are involved in the oxidative degradation of lignin, and 60 members of class AA6, which catalyze reductive degradation of aromatic compounds such as the monolignols that make up the lignin superstructure. Predicted protein sequences were also subject to BLAST analysis against the NCBI non-redundant (nr) database using BLASTp. In total 97,882 predicted proteins were matched to sequences in the $\mathrm{nr}$ database ( $~ 90 \%$ of total predictions). Using the KEGG extension of MEGAN4, over 32,000 functional associations were made to KEGG biochemical pathways from the BLAST output, of which 8,333 were attributed to carbohydrate metabolism. Multiple assignments to phosphotransferase systems (PTS) that facilitate internalization of many sugars in bacteria were also observed (Figure 2). These included 109 proteins that make up the three subunits of the PTS that facilitates specific internalization of cellobiose.

The gut CAZyme profile generated for $A$. ater was compared with those of humans, termites wallabies, giant pandas, and giant snails (Table 4). This comparison demonstrates that the number and proportion of cellulase-degrading enzymes in the slug gut are similar to what is found in both the snail and wallaby, with a similarly high number of oligosaccharide degrading enzymes in both molluscs. However, in the slug gut environment many more enzymes targeting hemicellulose were identified than in any of the comparator organisms. The SEED functional classifications of the microbiome were also compared to those of other gut environments, which demonstrated an increase in the proportion of genes involved in the processing of carbohydrates in the slug gut than in any comparator environment (Figure 3). This comparison also revealed that the SEED group representation in $A$. ater and the giant snail ( $A$. fulica) gut metagenomes were much more similar to each other than to the mammalian and insect comparator gut environments (Figure 3).

\section{Amplification and Expression of CAZymes}

To validate the metagenomic assembly and gene predictions, multiple genes were selected for amplification from the original metagenomic DNA sample. These included two full length predicted endocellulase genes, a full length $\beta$-glucosidase gene, a full length xylanase gene and a full length FAD-linked oxidase from the auxiliary activities 4 CAZyme group (Supplementary Datasets 3-5). As a proof of principal one partial gene was amplified (gene_id_77908) and subsequently extended to a full length gene using primers designed based on the top BLAST hit for that specific gene. Sanger sequencing of the resulting amplicons was carried out confirming amplification of the targeted predicted gene sequence. Five of six genes targeted were successfully amplified and full sequences confirmed. Gene 9459, a predicted $\beta$-glucosidase was also successfully amplified (Figure 4A), cloned and expressed in E. coli. The expression of a recombinant His-tagged protein of predicted size $(\sim 55 \mathrm{KDa})$ was confirmed using Western blotting (Figure 4B). The 9,459 strain was grown on a $\beta$-glucosidase activity growth plate (Figure $4 \mathrm{C}$ ) 


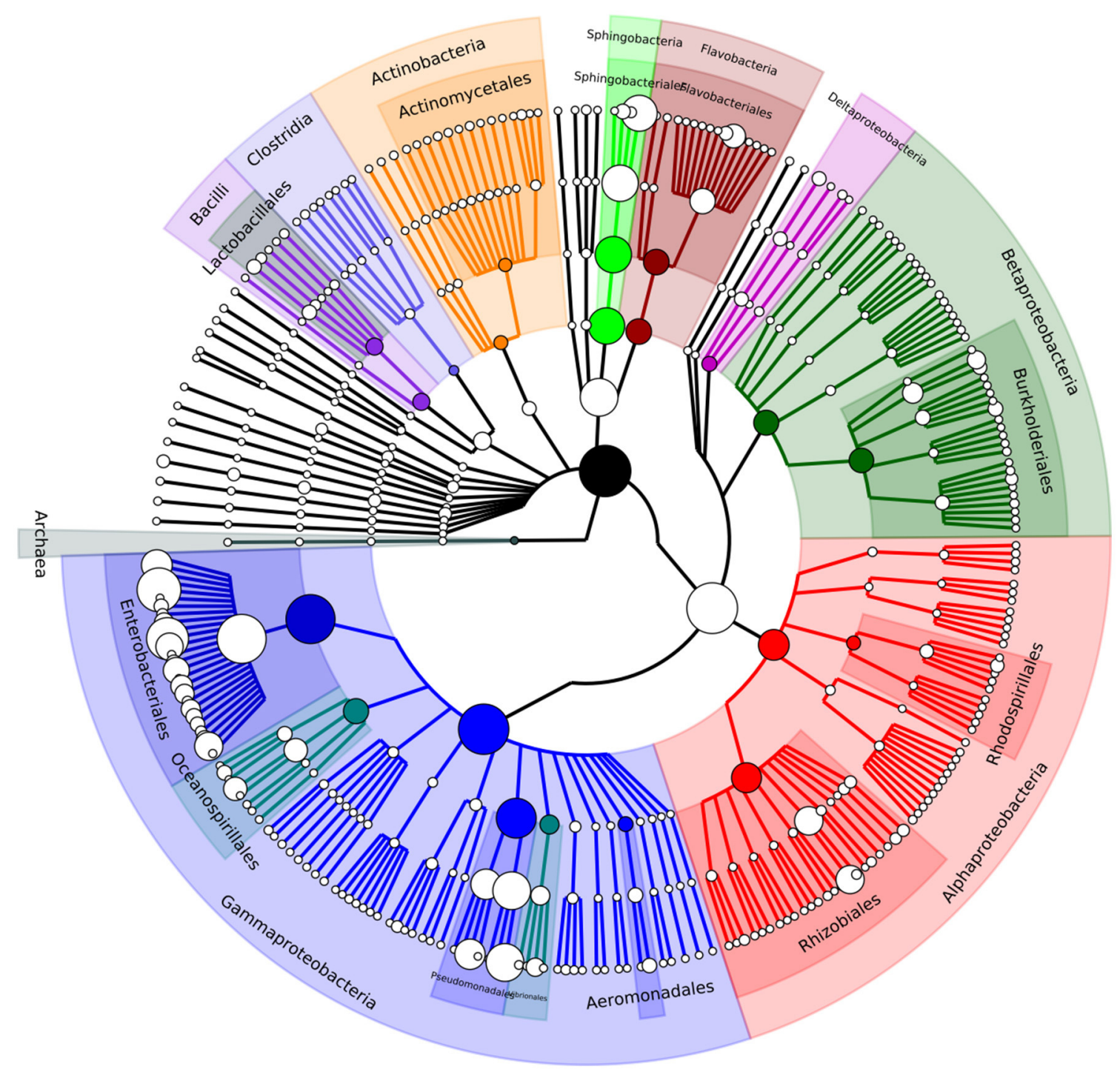

FIGURE 1 | A phylogenetic tree showing the diversity of the A. ater gut microbiome down to genus level. Visualised using GraPhIAn (Asnicar et al., 2015).

and tested positive for $\beta$-glucosidase. A control of untransformed TOP10 E. coli showed no activity on this assay.

\section{DISCUSSION}

The common black slug, A. ater has become one of the most widespread and successful Gastropod species in Europe and North America. The success of this (and other) species has caused the UK agricultural industry alone to spend almost $£ 30$ million each year on molluscicide pellets (Agular and Wink, 2005). Making it an important species in agro-economical terms.

Research into the digestive system of $A$. ater began in the 1960s, focusing on both carbohydrate breakdown (Evans and Jones, 1962) and protease activity (Evans and Jones, 1962). Further work determined rates of cellulose breakdown and characterized the $\mathrm{pH}$ and temperature profiles of gut fluids from black slugs of North American origin (James et al., 1997). In a previous study, we characterized the biochemical activity in the gut of the British black slug and identified multiple gut bacteria that exhibit cellulolytic activity. This work implicated the gut microbiome in the degradation of plant cell wall into simple sugars. In this study we tested the hypothesis that the slug gut microbiome could contribute to digestion and nutrient cycling, especially the breakdown of complex plant cell wall superstructures that are notoriously difficult for animals to degrade without substantial assistance from microbes (Hansen and Moran, 2014). This study has revealed an ecologically rich consortium of bacterial species in the $A$. ater gut that have previously been implicated in the digestion of tough vegetation. We have also demonstrated the vast metabolic repertoire that exists within the slug gut microbiome, including enzymes with potential to contribute to degradation of every major component 


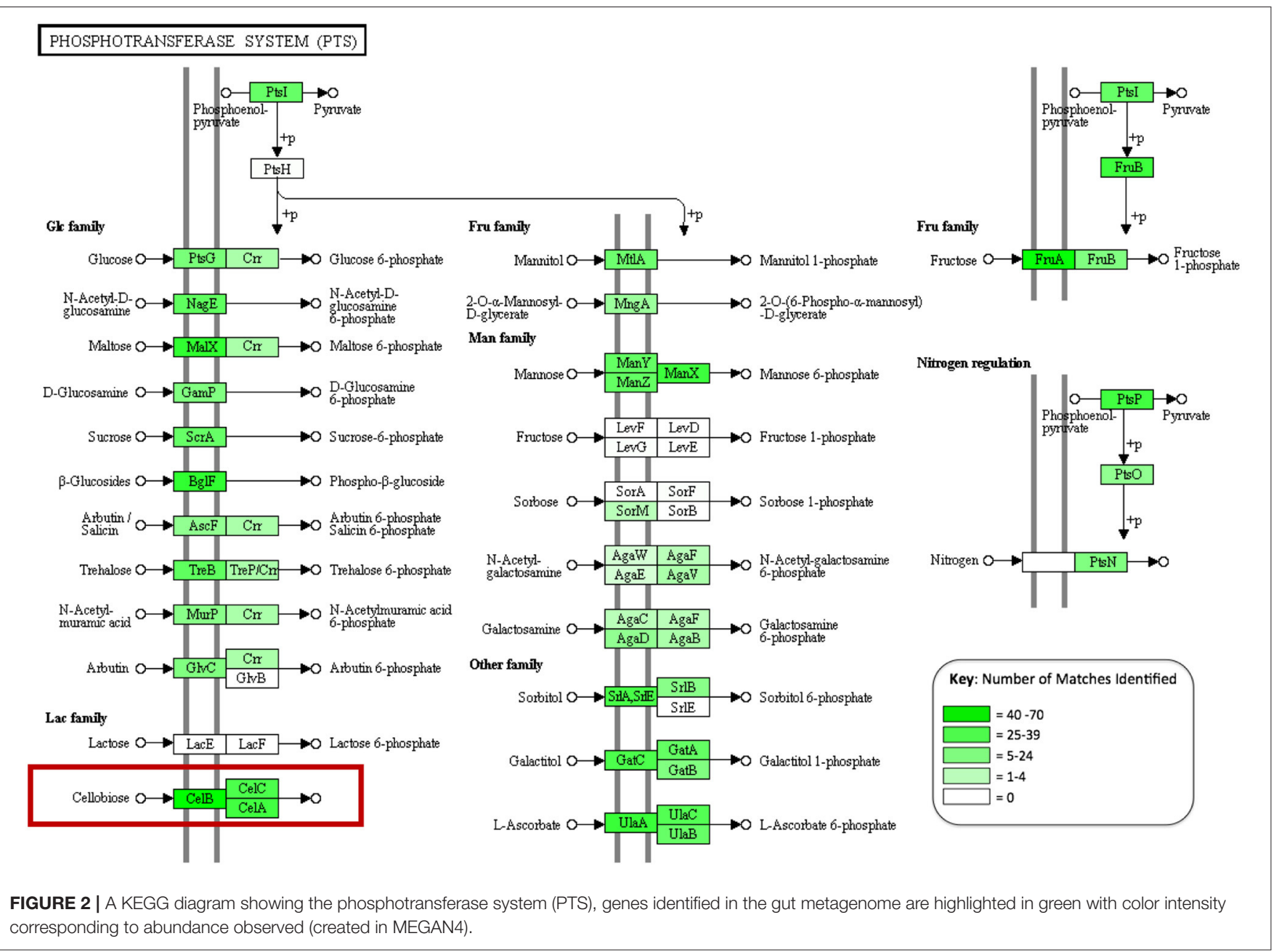

of plant cell wall superstructure, including lignin, which is widely considered to be the most difficult of these compounds to degrade enzymatically (Sanderson, 2011).

In total, Gammaproteobacteria accounted for the vast majority of the community metagenome, with $82 \%$ relative abundance; this included identification of 84 species in this class. The most abundant genera identified include Enterobacter, Citrobacter, Pseudomonas, Eschericia, Acinetobacter, and an unclassified genus belonging to the Sphingobacteriaceae family. These genera alone accounted for almost three quarters of the sequenced component of the gut metagenome (Table 2). Previous studies have shown dominance of the phylum Proteobacteria in gut microbiomes of various gastropod species, including freshwater planorbid snails (Biomphalaria pfeifferi) and terrestrial snails such as, the giant African land snail (A. fulica) (Cardoso et al., 2012b). Proteobacteria have also been seen to dominate other insect gut microbiomes whose diets are largely or entirely comprised of lignocellulose (Dillon and Dillon, 2004; Russell et al., 2009), which suggests a general association of this phylum not only with herbivorous insects but also with plant-eating gastropods. Furthermore, two studies of microbial consortia in fungal gardens used by leaf cutter ants (Atta colombica) to degrade lignocellulose both report dominance of the family Enterobacteriaceae (which account for $\sim 65 \%$ of the A. ater community metagenome) and predict this family to be directly involved in the efficient breakdown of plant material in these gardens (Suen et al., 2010; Aylward et al., 2012). A large number of genera were also detected in much lower abundances with over 200 genera account for only $\sim 27 \%$ of the microbiome, these may comprise transient elements of the gut microbiome that are ingested during proximal feeding or suppressed by nutritional cycling in the gut at a particular time. Our findings are also consistent with previous culture dependent identification of cellulolytic microbes from the $A$. ater gut, where almost all identifications made were in the Gammaproteobacteria class, and included many of the more abundant genera noted in this study (Joynson et al., 2014). These findings suggest that the gut environment of $A$. ater contains a consortium that is reflective of many highly efficient lignocellulose degrading environments.

Mining of the phylogenetic data associated with the gut microbiome identified several bacterial plant pathogens. These included six species recently ranked among the top 10 most important species of plant pathogen (Mansfield et al., 2012; 


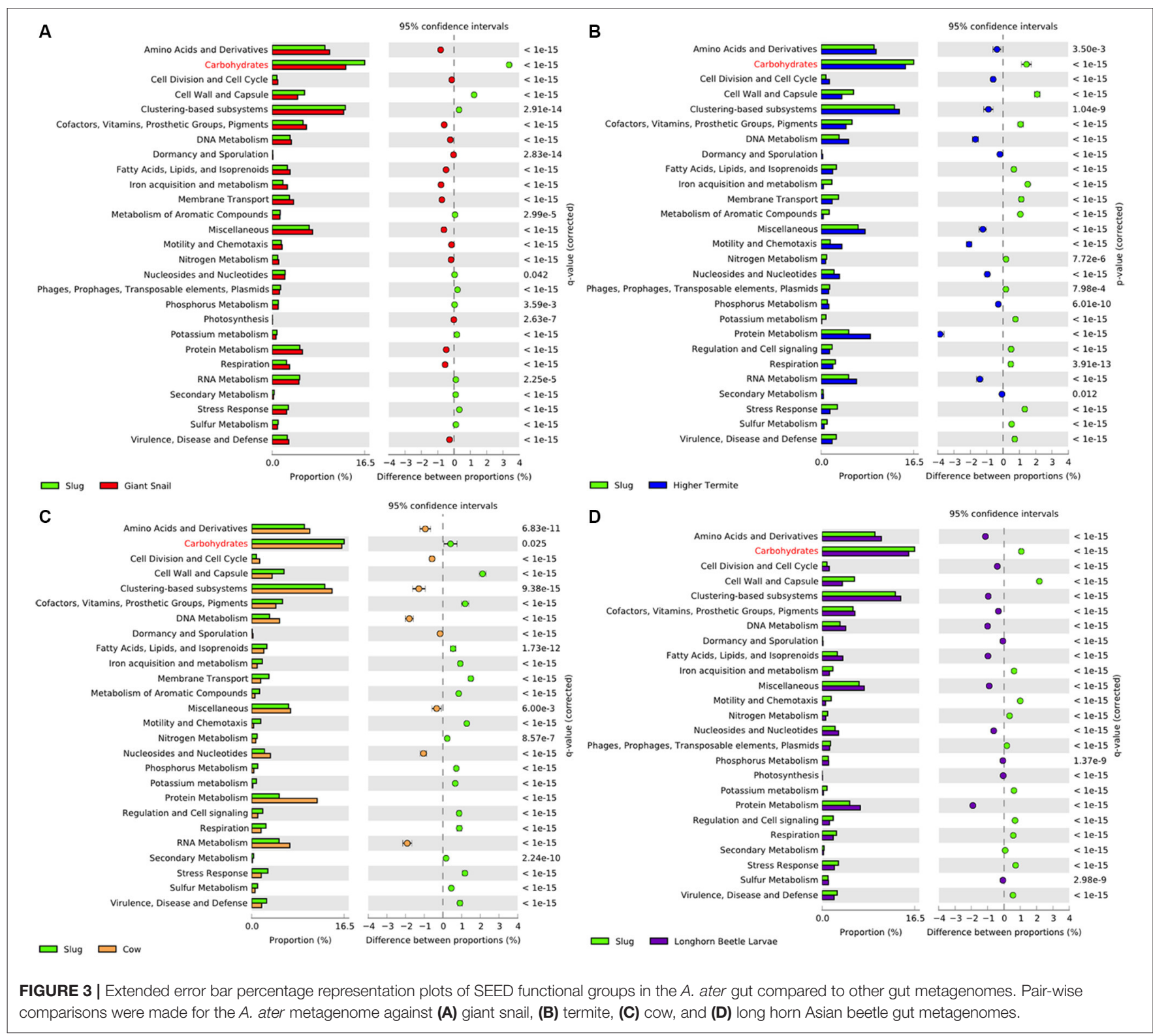

Table 3). Many of these pathogens are known to cause necrosis and eventual development of soft rot, blight, or blackleg in tuber based crops such as, potatoes, but also in ornamental plants and other crops. These include the three relatively closely related Enterobacteria Dickeya dadantii, P. carotovorum, and E. amylovora (Toth et al., 2011) with the latter two being identified previously in A. ater gut from samples taken in 2012 from the same area as this study (Joynson et al., 2014). If both of these pathogen species are commensally present in the slug gut, this would suggest that $A$. ater may act as a perpetual vector species through which they could be spread from field to field, and persist between growing seasons by overwintering in the slug gut. The role of insects in the transmission and overwintering of plant pathogens is now quite well established, the squash bug, flea beetle, and cucumber beetle are known to spread plant pathogens as well as sustaining populations of the pathogens they harbor during dormant winter months (Nadarasah and Stavrinides, 2011). However, more indepth study over multiple seasons would be required to confirm this hypothesis.

Functional analysis of the $A$. ater metagenome has yielded identification of 3,383 genes involved in the degradation of plant biomass, including all of the major components of the plant cell wall superstructure, cellulose, hemicellulose, and lignin supporting previous work that has implicated the slug gut microbiome in the facilitation of lignocellulose degradation (James et al., 1997). The largest proportion of these (641) breakdown oligosaccharides, including $204 \beta$-glucosidases, 80 $\beta$-galactosidases, and $279 \beta$-xylosidases. Numbers of long chain carbohydrate degrading enzymes were lower in comparison, 


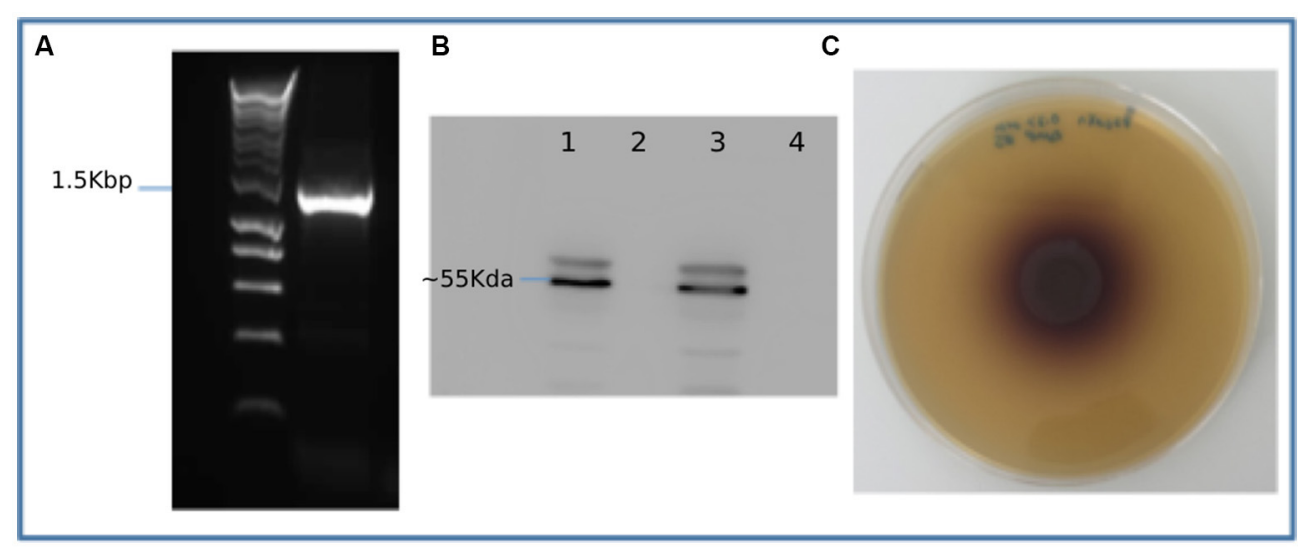

FIGURE 4 | Recombinant expression and activity testing of gene 9459: (A) Amplification of gene 9459 (B) A Western blot showing successful expression of recombinant protein lanes 1 and 3 showing duplicate induced samples and lanes 2 and 4 showing duplicate negative controls (C) An esculin hydrate- ferric ammonium citrate activity plate showing the gene 9459 clone $\beta$-glucosidase activity.

TABLE 2 | A selection of the most abundant phylogenetic groups present in the gut microbial community down to genus level.

\begin{tabular}{|c|c|}
\hline Classification & Percentage abundance (\%) \\
\hline k__Bacteria & 99.99 \\
\hline k__Archaea & 0.01 \\
\hline p__Proteobacteria & 88.15 \\
\hline c__Gammaproteobacteria & 82.16 \\
\hline o__Enterobacteriales & 64.56 \\
\hline f_Enterobacteriaceae & 64.56 \\
\hline g_Enterobacter & 26.86 \\
\hline g_Citrobacter & 19.86 \\
\hline g_Escherichia & 3.91 \\
\hline O_Pseudomonadales & 14.25 \\
\hline f__Pseudomonadaceae & 10.56 \\
\hline g_Pseudomonas & 10.54 \\
\hline f__Moraxellaceae & 3.69 \\
\hline g_Acinetobacter & 3.68 \\
\hline p_Bacteroidetes & 10.53 \\
\hline c__Sphingobacteria & 8.57 \\
\hline o__Sphingobacteriales & 8.57 \\
\hline f__Sphingobacteriaceae & 8.56 \\
\hline g__Sphingobacteriaceae_unclassified & 8.10 \\
\hline p_Firmicutes & 0.59 \\
\hline p_Actinobacteria & 0.28 \\
\hline p_Chlamydiae & 0.21 \\
\hline p_Chloroflexi & 0.16 \\
\hline
\end{tabular}

Phylogenetic classifications and microbial abundance estimations were made using MetaPhIAn to compare sequences to a clade specific marker database.

with only 26 cellulase enzymes being identified in total. The dominance of oligosaccharide degrading enzymes appears in all of the other comparator gut environments shown in Table 4, including wallabies, termites, and also in the gut microbiomes of reindeer and cattle (Pope et al., 2012) with similar patterns
TABLE 3 | Microbiome abundance of plant pathogens present in the $A$. ater gut microbiome, as ranked by a survey of experts carried out by Mansfield et al. (2012).

\begin{tabular}{llc}
\hline Ranking & Pathogenic species & Microbiome abundance (\%) \\
\hline 1 & Pseudomonas syringae & 0.08264 \\
3 & Agrobacterium tumefaciens & 0.06987 \\
5 & Xanthomonas campestris & 0.0144 \\
7 & Enwinia amylovora & 0.03587 \\
9 & Dickeya dadantii & 0.04896 \\
10 & Pectobacterium carotovorum & 0.04215 \\
\hline
\end{tabular}

also observed in environmental microbiomes such as, leaf cutter ant fungus gardens (Aylward et al., 2012). This could support the hypothesis that gut microbes are predominantly involved in the breakdown of partially degraded plant material (be it partially rotten when ingested or chemically pre-processed in a stomach) across the board. However, there is still the possibility that some groups of microbial lignocellulose degrading enzymes that are unknown and may be undetectable using homology-based methods. Enzyme groups that are involved in the degradation of hemicellulose are seen in especially high numbers in the $A$. ater gut when compared with other gut microbiomes, with larger numbers for both the degradation long chain hemicellulose (321) and its derived oligosaccharides (437). Further indications that sugars in plant cell walls are utilized by gut microbes come from the identification of numerous sugar transporter proteins. These include a large number of components of the cellobiose-specific PTS that facilitate the uptake of cellulose degradation products (Figure 2). The KEGG diagram in Figure 2 also shows the presence of membrane transport system components specific to mannose and $\beta$-glucosides. Together, the identification of multiple enzymes that break down plant cell walls and the transport systems that facilitate the uptake of the resulting oligosaccharides provide a strong indication that the microbial population has an active role in the 
TABLE 4 | Comparison of the glycoside hydrolase (GH) profiles of human, termite, wallaby, giant panda, snail, and slug gut metagenomes as classified by Cardoso et al. (2012a) and Allgaier et al. (2010), showing GH groups that are involved in the breakdown/modification of plant cell wall polysaccharides.

\begin{tabular}{|c|c|c|c|c|c|c|c|}
\hline Pfam group & Predominant activity & Human & Termite & Wallaby & Panda & Snail & Slug \\
\hline GH5 & Cellulases & 7 & 125 & 27 & 1 & 36 & 15 \\
\hline $\mathrm{GH} 6$ & Endoglucanases & 0 & 0 & 0 & 0 & 4 & 0 \\
\hline $\mathrm{GH} 7$ & Endoglucanases & 0 & 0 & 0 & 0 & 0 & 0 \\
\hline $\mathrm{GH} 9$ & Endoglucanases & 0 & 43 & 5 & 0 & 15 & 11 \\
\hline $\mathrm{GH} 44$ & Endoglucanases & 0 & 0 & 0 & 0 & 0 & 0 \\
\hline GH45 & Endoglucanases & 0 & 6 & 0 & 0 & 0 & 0 \\
\hline GH48 & Cellobiohydrolases & 0 & 0 & 0 & 0 & 2 & 0 \\
\hline Total & & 7 & 174 & 32 & 1 & 57 & 26 \\
\hline \multicolumn{8}{|c|}{ ENDOHEMICELLULASES } \\
\hline $\mathrm{GH} 8$ & Endoxylanases & 2 & 21 & 2 & 1 & 46 & 11 \\
\hline GH10 & Endo-1,4- $\beta$-xylanase & 2 & 102 & 19 & 1 & 25 & 16 \\
\hline GH11 & Xylanase & 0 & 19 & 0 & 0 & 1 & 0 \\
\hline GH12 & Endoglucanase \& xyloglucanase & 0 & 0 & 0 & 0 & 0 & 12 \\
\hline GH26 & $\beta$-mannanase \& xylanase & 1 & 20 & 8 & 0 & 11 & 0 \\
\hline $\mathrm{GH} 28$ & Galacturonases & 3 & 15 & 10 & 0 & 69 & 6 \\
\hline GH53 & Endo-1,4- $\beta$-galactanase & 11 & 20 & 11 & 4 & 9 & 276 \\
\hline Total & & 19 & 197 & 50 & 6 & 161 & 321 \\
\hline \multicolumn{8}{|c|}{ XYLOGLUCANASES } \\
\hline GH16 & Xyloglucanases & 1 & 6 & 6 & 6 & 12 & 117 \\
\hline $\mathrm{GH} 17$ & 1,3- $\beta$-glucosidases & 0 & 0 & 0 & 0 & 2 & 60 \\
\hline GH81 & $1,3-\beta$-glucanases & 0 & 0 & 0 & 0 & 1 & 0 \\
\hline Total & & 1 & 6 & 6 & 6 & 15 & 177 \\
\hline \multicolumn{8}{|c|}{ DEBRANCHING ENZYMES } \\
\hline GH51 & $\alpha$-L-arabinofuranosidases & 15 & 13 & 19 & 2 & 22 & 3 \\
\hline GH62 & $\alpha$-L-arabinofuranosidases & 0 & 0 & 0 & 0 & 2 & 0 \\
\hline GH67 & $\alpha$-glucuronidase & 1 & 6 & 1 & 2 & 5 & 1 \\
\hline GH78 & $\alpha$-L-rhmnosidase & 13 & 7 & 46 & 1 & 73 & 8 \\
\hline Total & & 29 & 26 & 66 & 5 & 102 & 12 \\
\hline \multicolumn{8}{|c|}{ OLIGOSACCHARIDE DEGRADING ENZYMES } \\
\hline $\mathrm{GH} 1$ & Mainly $\beta$-glucosidases & 54 & 27 & 94 & 41 & 294 & 118 \\
\hline $\mathrm{GH} 2$ & Mainly $\beta$-galactosidases & 29 & 32 & 39 & 4 & 66 & 60 \\
\hline $\mathrm{GH} 3$ & Mainly $\beta$-glucosidases & 55 & 109 & 101 & 11 & 219 & 86 \\
\hline GH29 & $\alpha$-L-fucosidases & 7 & 12 & 5 & 0 & 70 & 11 \\
\hline GH35 & $\beta$-galactosidase & 4 & 7 & 8 & 1 & 32 & 14 \\
\hline GH38 & $\alpha$-mannosidase & 6 & 18 & 3 & 8 & 18 & 39 \\
\hline GH39 & $\beta$-xylosidase & 2 & 13 & 3 & 8 & 6 & 279 \\
\hline $\mathrm{GH} 42$ & $\beta$-galactosidases & 15 & 33 & 17 & 7 & 54 & 6 \\
\hline $\mathrm{GH} 43$ & Arabinases \& xylosidases & 34 & 63 & 72 & 13 & 185 & 28 \\
\hline GH52 & $\beta$-xylosidase & 0 & 3 & 0 & 0 & 0 & 0 \\
\hline Total & & 206 & 317 & 342 & 93 & 944 & 641 \\
\hline
\end{tabular}

extracellular breakdown of plant cell wall components in the A. ater gut.

Several predicted genes from this metagenome were successfully amplified from whole genome amplified gut metagenomic DNA, confirmed by Sanger sequencing. This validates the assembly and the predictions made thereof, showing that it is very likely that the predicted sequences do exist in nature. We then successfully expressed a full length predicted $\beta$-glucosidase gene and observed the enzymatic function using growth plate assays. To our knowledge we are the first to succeed in amplifying novel, functioning genes from a whole genome amplified metagenomic sample. The use of whole genome amplified samples enables studies of a far greater number of predicted genes by sidestepping the problem of small sample size often seen with environmental samples, which limits the scope for genes of interest to be studied using expensive gene synthesis methods.

The use of metagenomics in the study of environmental DNA offers a new means to advance our knowledge of microbial 
communities. Here we use metagenomics to gain an insight into both the phylogeny and the functional capability of the gut microbiome of the common black slug. This work demonstrates that the microbial community is dominated by a relatively low number of genera with the Enterobacter genus being observed in especially high numbers. This study also implicates the slug gut microbiome in the degradation of lignocellulose. Here we identified a large repertoire of genes that offer potential for lignocellulose not only to be degraded but also for the resulting sugars to be taken up by members of the microbiome itself. We have also validated our predictions through amplification of selected glycoside hydrolase genes along with observing predicted functional activity in of an amplified $\beta$-glucosidase gene. Our work therefore begins to shed light on how the black slug can process the large quantities of plant biomass it consumes and provides a further example of a herbivore gut microbiome which is well equipped to breakdown plant matter. In addition, by identifying plant pathogen species harbored in the gut we raise questions as to the potential role of the slug in the transmission and wintering of pathogen species. This knowledge is of considerable potential relevance following the 2014 European Union wide ban on the use of some traditional molluscicide pellets in agriculture.

\section{DATA AVAILABILITY}

Sequence data from this project has been uploaded to EBI under project number PRJEB21599 (http://www.ebi.ac.uk/ena/ data/view/PRJEB21599).

\section{REFERENCES}

Agular, R., and Wink, M. (2005). How do slugs cope with toxic alkaloids? Chemoecology 15, 167-177. doi: 10.1007/s00049-005-0309-5

Allgaier, M., Reddy, A., Park, J., Ivanova, N., D’Haeseleer, P., Lowry, S., et al. (2010). Targeted discovery of glycoside hydrolases from a switchgrassadapted compost community. PLoS ONE 5:e8812. doi: 10.1371/journal.pone.00 08812

Andrews, S. (2010). FastQC A Quality Control tool for High Throughput Sequence Data. Available online at: http://www.bioinformatics.babraham.ac.uk/projects/ fastqc/

Asnicar, F., Weingart, G., Tickle, T. L., Huttenhower, C., and Segata, N. (2015). Compact graphical representation of phylogenetic data and metadata with GraPhlAn. Peer J. 3:e1029. doi: 10.7717/peerj.1029

Aylward, F. O., Burnum, K. E., Scott, J. J., Suen, G., Tringe, S. G., Adams, S. M., et al. (2012). Metagenomic and metaproteomic insights into bacterial communities in leaf-cutter ant fungus gardens. ISME J. 6, 1688-1701. doi: 10.1038 /ismej.2012.10

Bertino-Grimaldi, D., Medeiros, M. N., Vieira, R. P., Cardoso, A. M., Turque, A. S., Silveira, C. B., et al. (2013). Bacterial community composition shifts in the gut of Periplaneta americana fed on different lignocellulosic materials. Springerplus 2:609. doi: 10.1186/2193-1801-2-609

Brune, A. (2014). Symbiotic digestion of lignocellulose in termite guts. Nat. Rev. Microbiol. 12, 168-180. doi: 10.1038/nrmicro3182

Cardoso, A. M., Cavalcante, J. J., Cantao, M. E., Thompson, C. E., Flatschart, R. B., Glogauer, A., et al. (2012a). Metagenomic analysis of the microbiota from the crop of an invasive snail reveals a rich reservoir of novel genes. PLOS ONE 7:e48505. doi: 10.1371/journal.pone.0048505

\section{ETHICS STATEMENT}

As this study uses only invertebrates ( $A$. ater), the UK and EU ethics directives for animal testing do not apply. With EU DIRECTIVE 2010/63/EU ON PROTECTION OF ANIMALS USED FOR SCIENTIFIC PURPOSES applying to only "live non-human vertebrate animals" and "live cephalopods."

\section{AUTHOR CONTRIBUTIONS}

Conceptualization of the project: NF and RJ; Sample collection: RJ; DNA extraction and Molecular Biology: RJ and EO; Bioinformatics analyses: RJ and LP; Data interpretation: RJ and LP; Manuscript writing, RJ and NF with contributions from LP, EO.

\section{ACKNOWLEDGMENTS}

We thank The Centre for Genomic Research staff for their sequencing expertise particularly G. Weedall and R. Chaudhuri for their bioinformatics advice. Thanks also to Dominic Wood and John Gatehouse for access to computing recourses. We would also like to thank the University of Salford Innovation fund for supporting this research.

\section{SUPPLEMENTARY MATERIAL}

The Supplementary Material for this article can be found online at: https://www.frontiersin.org/articles/10.3389/fmicb. 2017.02181/full\#supplementary-material

Cardoso, A. M., Cavalcante, J. J. V., Vieira, R. P., Lima, J. L., Grieco, M. A. B., Clementino, M. M., et al. (2012b). Gut bacterial communities in the giant land snail Achatina fulica and their modification by sugarcane-based diet. PLOS ONE 7:e33440. doi: 10.1371/journal.pone.0033440

Dillon, R. J., and Dillon, V. M. (2004). The gut bacteria of insects: nonpathogenic interactions. Annu. Rev. Entomol. 49, 71-92. doi: 10.1146/annurev.ento.49.061802.123416

Evans, W. A. L., and Jones, E. G. (1962). A note on the proteinase activity in the alimentary tract of the slug Arion ater L. Comp. Biochem. Physiol. 5, 223-225. doi: 10.1016/0010-406X(62)90108-1

Gismervik, K., Bruheim, T., Rorvik, L. M., Haukeland, S., and Skaar, I. (2014). Invasive slug populations (Arion vulgaris) as potential vectors for Clostridium botulinum. Acta Vet. Scand. 56:65. doi: 10.1186/s13028-014-0065-z

Hansen, A. K., and Moran, N. A. (2014). The impact of microbial symbionts on host plant utilization by herbivorous insects. Mol. Ecol. 23, 1473-1496. doi: $10.1111 / \mathrm{mec} .12421$

Hess, M., Sczyrba, A., Egan, R., Kim, T. W., Chokhawala, H., Schroth, G., et al. (2011). Metagenomic discovery of biomass-degrading genes and genomes from cow rumen. Science 331, 463-467. doi: 10.1126/science.1200387

Huson, D. H., Mitra, S., Ruscheweyh, H. J., Weber, N., and Schuster, S. C. (2011) Integrative analysis of environmental sequences using MEGAN4. Genome Res. 21, 1552-1560. doi: 10.1101/gr.120618.111

Ireland, M. P. (1979). Distribution of essential and toxic metals in the Terrestrial gastropod arion-ater. Environ. Pollut. 20, 271-278. doi: 10.1016/0013-9327(79)90150-2

James, R., Nguyen, T., Arthur, W., Levine, K., and Williams, D. C. (1997) Hydrolase (beta-glucanase, alpha-glucanase, and protease) activity in Ariolimax columbianus (banana slug) and Arion ater (garden 
slug). Comp. Biochem. Physiol. B Biochem. Mol. Biol. 118, 275-283. doi: 10.1016/S0305-0491(97)00058-8

Joynson, R., Swamy, A., Bou, P. A., Chapuis, A., and Ferry, N. (2014). Characterization of cellulolytic activity in the gut of the terrestrial land slug Arion ater: biochemical identification of targets for intensive study. Comp. Biochem. Physiol. B Biochem. Mol. Biol. 177-178, 29-35. doi: 10.1016/j.cbpb.2014.08.003

Kay, P., and Grayson, R. (2013). Using water industry data to assess the metaldehyde pollution problem. Water Environ. J. 28, 410-417. doi: $10.1111 /$ wej.12056

Kozlowski, J. (2007). The distribution, biology, population dynamics and harmfulness of Arion lusitanicus Mabille, 1868 (Gastropoda: Pulmonata: Arionidae) in Poland. J. Plant Protect. Res. 47, 219-230.

Langmead, B., and Salzberg, S. L. (2012). Fast gapped-read alignment with Bowtie 2. Nat. Methods 9, 357-359. doi: 10.1038/nmeth.1923

Levasseur, A., Drula, E., Lombard, V., Coutinho, P. M., and Henrissat, B. (2013). Expansion of the enzymatic repertoire of the CAZy database to integrate auxiliary redox enzymes. Biotechnol. Biofuels 6:41. doi: 10.1186/1754-6834-6-41

Li, H., Handsaker, B., Wysoker, A., Fennell, T., Ruan, J., Homer, N., et al. (2009). The sequence alignment/map format and SAMtools. Bioinformatics 25, 2078-2079. doi: 10.1093/bioinformatics/btp352

Lombard, V., Ramulu, H. G., Drula, E., Coutinho, P. M., and Henrissat, B. (2014). The carbohydrate-active enzymes database (CAZy) in 2013. Nucleic Acids Res. 42, D490-D495. doi: 10.1093/nar/gkt1178

Mansfield, J., Genin, S., Magori, S., Citovsky, V., Sriariyanum, M., Ronald, P., et al. (2012). Top 10 plant pathogenic bacteria in molecular plant pathology. Mol. Plant Pathol. 13, 614-629. doi: 10.1111/j.1364-3703.2012.00804.x

Meyer, F., Paarmann, D., D’Souza, M., Olson, R., Glass, E. M., Kubal, M., et al. (2008). The metagenomics RAST server - a public resource for the automatic phylogenetic and functional analysis of metagenomes. BMC Bioinformatics 9:386. doi: 10.1186/1471-2105-9-386

Milne, I., Stephen, G., Bayer, M., Cock, P. J. A., Pritchard, L., Cardle, L., et al. (2013). Using Tablet for visual exploration of second-generation sequencing data. Brief. Bioinformatics 14, 193-202. doi: 10.1093/bib/bbs012

Nadarasah, G., and Stavrinides, J. (2011). Insects as alternative hosts for phytopathogenic bacteria. FEMS Microbiol. Rev. 35, 555-575. doi: 10.1111/j.1574-6976.2011.00264.x

Namiki, T., Hachiya, T., Tanaka, H., and Sakakibara, Y. (2012). MetaVelvet: an extension of Velvet assembler to de novo metagenome assembly from short sequence reads. Nucleic Acids Res. 40:e155. doi: 10.1093/nar/gks678

Park, B. H., Karpinets, T. V., Syed, M. H., Leuze, M. R., and Uberbacher, E. C. (2010). CAZymes Analysis Toolkit (CAT): web service for searching and analyzing carbohydrate-active enzymes in a newly sequenced organism using CAZy database. Glycobiology 20, 1574-1584. doi: 10.1093/glycob/cwq106

Parks, D. H., Tyson, G. W., Hugenholtz, P., and Beiko, R. G. (2014). STAMP: statistical analysis of taxonomic and functional profiles. Bioinformatics 30, 3123-3124. doi: 10.1093/bioinformatics/btu494
Pope, P. B., Mackenzie, A. K., Gregor, I., Smith, W., Sundset, M. A., McHardy, A. C., et al. (2012). Metagenomics of the Svalbard reindeer rumen microbiome reveals abundance of polysaccharide utilization loci. PLOS ONE 7:e38571. doi: 10.1371/journal.pone.0038571

Qin, J. J., Li, R. Q., Raes, J., Arumugam, M., Burgdorf, K. S., Manichanh, C., et al. (2010). A human gut microbial gene catalogue established by metagenomic sequencing. Nature 464, 59-65. doi: 10.1038/nature08821

Russell, J. A., Moreau, C. S., Goldman-Huertas, B., Fujiwara, M., Lohman, D. J., and Pierce, N. E. (2009). Bacterial gut symbionts are tightly linked with the evolution of herbivory in ants. Proc. Natl. Acad. Sci. U.S.A. 106, 21236-21241. doi: 10.1073/pnas.0907926106

Sanderson, K. (2011). Lignocellulose: a chewy problem. Nature 474, S12-S14. doi: $10.1038 / 474$ S012a

Segata, N., Waldron, L., Ballarini, A., Narasimhan, V., Jousson, O., and Huttenhower, C. (2012). Metagenomic microbial community profiling using unique clade-specific marker genes. Nat. Methods 9, 811-814. doi: $10.1038 /$ nmeth.2066

Seric Jelaska, L., Jurasovic, J., Brown, D. S., Vaughan, I. P., and Symondson, W. O. (2014). Molecular field analysis of trophic relationships in soil-dwelling invertebrates to identify mercury, lead and cadmium transmission through forest ecosystems. Mol. Ecol. 23, 3755-3766. doi: 10.1111/mec.12566

Slotsbo, S., Hansen, L. M., and Holmstrup, M. (2011). Low temperature survival in different life stages of the Iberian slug, Arion lusitanicus. Cryobiology 62, 68-73. doi: 10.1016/j.cryobiol.2010.12.005

Suen, G., Scott, J. J., Aylward, F. O., Adams, S. M., Tringe, S. G., Pinto-Tomás, A. A., et al. (2010). An insect herbivore microbiome with high plant biomass-degrading capacity. PLoS Genet. 6:e1001129. doi: 10.1371/journal.pgen.1001129

Toth, I. K., van der Wolf, J. M., Saddler, G., Lojkowska, E., Hélias, V., Pirhonen, M., et al. (2011). Dickeya species: an emerging problem for potato production in Europe. Plant Pathol. 60, 385-399. doi: 10.1111/j.1365-3059.2011.02427.x

Zerbino, D. R., and Birney, E. (2008). Velvet: algorithms for de novo short read assembly using de Bruijn graphs. Genome Res. 18, 821-829. doi: 10.1101 /gr.074492.107

Zhu, W., Lomsadze, A., and Borodovsky, M. (2010). Ab initio gene identification in metagenomic sequences. Nucleic Acids Res. 38:e132. doi: 10.1093/nar/gkq275

Conflict of Interest Statement: The authors declare that the research was conducted in the absence of any commercial or financial relationships that could be construed as a potential conflict of interest.

Copyright (C) 2017 Joynson, Pritchard, Osemwekha and Ferry. This is an open-access article distributed under the terms of the Creative Commons Attribution License (CC $B Y)$. The use, distribution or reproduction in other forums is permitted, provided the original author(s) or licensor are credited and that the original publication in this journal is cited, in accordance with accepted academic practice. No use, distribution or reproduction is permitted which does not comply with these terms. 